Article

\title{
Generalized Weyl-Heisenberg Algebra, Qudit Systems and Entanglement Measure of Symmetric States via Spin Coherent States. Part II: The Perma-Concurrence Parameter
}

\author{
Mohammed Daoud ${ }^{1,2}$ and Maurice R. Kibler 2,3,4,*(D) \\ 1 Department of Physics, Faculty of Sciences, University Ibn Tofail, BP 133 Kénitra, Morocco \\ 2 CNRS/IN2P3, Institut de Physique Nucléaire, 69622 Villeurbanne, France \\ 3 Faculté des Sciences et Technologies, Université Claude Bernard Lyon 1, 69622 Villeurbanne, France \\ 4 IDEXLYON, Université de Lyon, 69361 Lyon, France \\ * Correspondence: m.kibler@ipnl.in2p3.fr
}

Received: 14 June 2019; Accepted: 27 June 2019; Published: 3July 2019

\begin{abstract}
This paper deals with separable and entangled qudits $\left|\psi_{d}\right\rangle$ (quantum states in dimension $d$ ) constructed from Dicke states made of $N=d-1$ qubits. Such qudits present the property to be totally symmetric under the interchange of the $N$ qubits. We discuss the notion of perma-concurrence $P_{d}$ for the qudit $\left|\psi_{d}\right\rangle$, introduced by the authors (Entropy 2018, 20, 292), as a parameter for characterizing the entanglement degree of $\left|\psi_{d}\right\rangle$. For $d=3$, the perma-concurrence $P_{3}$ constitutes an alternative to the concurrence $C$ for symmetric two-qubit states. We give several expressions of $P_{d}$ (in terms of matrix permanent and in terms of unit vectors of $\mathbb{R}^{3}$ pointing on the Bloch sphere) and precise the range of variation of $P_{d}$ (going from separable to maximally entangled states). Numerous examples are presented for $P_{d}$. Special attention is devoted to states of $\mathrm{W}$ type and to maximally entangled states of Bell and Greenberger-Horne-Zeilinger type.
\end{abstract}

Keywords: generalized Weyl-Heisenberg algebra; qubits and qudits; Dicke states; SU(2) coherent states; entanglement; concurrence; perma-concurrence; Majorana stars

\section{Introduction}

Entanglement in multi-qubit systems is a valuable resource in implementing several quantum protocols offering significant advantages in the communication and processing of information [1]. Quantum teleportation [2,3] and quantum cryptography or quantum key distribution [4] constitute promising applications in quantum information science.

The characterisation, classification and identification of genuine entanglement in multipartite quantum systems remain a challenging issue and continue presently to attract attention in the field of quantum information theory. Several measures of quantum correlations in bipartite and multipartite quantum systems have been introduced in the literature [5-8]. The Wootters concurrence and the entanglement of formation are two examples of quantitative measures of entanglement [9]; the concurrence is a simple tool to characterize the degree of entanglement of two-qubit systems in pure states. Another indicator of quantum correlations in bipartite quantum systems is the quantum discord based on the von Neumann entropy. This parameter was introduced by Ollivier and Zurek [10] and by Henderson and Vedral [11]; it goes beyond entanglement and provide a proper tool to investigate the quantum correlations in an arbitrary bipartite state even for a separable state. In general, the analytical evaluation of the quantum discord is difficult and only partial results were obtained for some special two-qubit states. 
Unfortunately, there is no simple way of measuring entanglement for multi-qubit systems. It is thus of paramount importance to find an adequate formalism to understand the genuine entanglement in multi-qubit systems. The first attempt to find a measure of three-qubit entanglement was made by Coffmann et al. by introducing the notion of 3-tangle [12]. This idea was extended to $N$-tangle by Wong and Christensen [13] for an even number $N$ of qubits. Clearly, developing the tools to distinguish between different classes of multipartite entangled states is essential in quantum information theory. In this respect, the classification of mutli-entangled states was approached via local unitary operations, local operations and classical communication, and stochastic local operations assisted by classical communication (see the list of references quoted in [14]). Another way to deal with entanglement in multi-qubit systems consists of using the entanglement witness method [7,15]. Despite the intensive efforts dedicated to this problem, the determination of quantum correlations in multi-qubit states remains a highly non-trivial issue in entanglement theory. Even though several tools and parameters were introduced, it remains difficult in several cases to decide between entanglement and separability of a given multipartite quantum state. This problem was shown to belong to the complexity class NP in the dimension of the quantum system.

In this paper, we shall use the notion of the perma-concurrence $P_{d}$ introduced in [16] to deal with the entanglement of a state $\left|\psi_{d}\right\rangle$ involving $N=d-1(d \geq 3)$ qubits, state invariant under the group $S_{N}$. The paper is organized as follows. Section 2 is concerned with some generalities (generalized Weyl-Heisenberg algebra, Dicke states and Majorana states). The perma-concurrence $P_{d}$ is studied with numerous examples in Section 3. Sections 4 and 5 are devoted to the perma-concurrence for states of $W$ type and states of Bell and Greenberger-Horne-Zeilinger (GHZ) type, respectively. Some conclusions are given in a last section.

In the present article, we use the following notations: $A^{\dagger},[A, B], \mathbb{I}, \delta_{i, j}$, and $C_{N}^{k}$ stand for the adjoint of the operator $A$, the commutator of the operators $A$ and $B$, the identity operator, the Kronecker $\delta$, and the binomial coefficient, respectively.

\section{Generalities}

In this section we briefly review, in a quantum information context, the main ingredients of the generalized Weyl-Heisenberg algebra $[17,18]$ for which the Dicke states [16,19-21] and the Majorana states [22] play a central role. The interest of Dicke states and Majorana states is important for quantum information. For instance, some particular classes of Dicke states are known to be decoherence free or noiseless under the environment effects [23]. This important property makes this kind of states very useful in encoding quantum information in qudit systems [24]. Furthermore, it has been mentioned that the Majorana representation of Dicke states can be of special interest in investigating and understanding decoherence free or noiseless multi-qubit systems [25].

\subsection{Generalized Weyl-Heisenberg Algebra}

Let $\mathcal{H}_{2}$ be the two-dimensional Hilbert space with an orthonormal basis $\{|0\rangle,|1\rangle\}$. In quantum mechanics, the vectors $|0\rangle$ and $|1\rangle$ can represent the states for a two-level system (e.g., $|0\rangle=\left|\frac{1}{2}, \frac{1}{2}\right\rangle$ and $|1\rangle=\left|\frac{1}{2},-\frac{1}{2}\right\rangle$ for a $\frac{1}{2}$-spin particle); the states $|0\rangle$ and $|1\rangle$ are then refereed to as qubits. The operators

$$
q^{-}=|0\rangle\left\langle 1\left|, \quad q^{+}=\right| 1\right\rangle\langle 0|, \quad K=| 1\rangle\langle 1|
$$

satisfy the relations

$$
\left(q^{-}\right)^{+}=q^{+}, \quad K^{+}=K, \quad\left[q^{-}, q^{+}\right]=\mathbb{I}-2 K, \quad\left[K, q^{+}\right]=q^{+}, \quad\left[K, q^{-}\right]=-q^{-}
$$

The $2^{N}$-dimensional Hilbert space $\mathcal{H}_{2^{N}}$ for a system of $N$ qubits is made of linear combinations of vectors belonging to

$$
\mathcal{H}_{2} \otimes \mathcal{H}_{2} \otimes \cdots \otimes \mathcal{H}_{2}
$$


with $N$ factors. Let us consider the set $\left\{q_{i}^{-}, q_{i}^{+}, K_{i}: i=1,2, \cdots, N\right\}$ of operators acting on $\mathcal{H}_{2^{N}}$. As an extension of Equation (1), we take

$$
\begin{gathered}
\left(q_{i}^{-}\right)^{+}=q_{i}^{+}, \quad K_{i}^{+}=K_{i}, \quad\left[q_{i}^{-}, q_{j}^{-}\right]=\left[q_{i}^{+}, q_{j}^{+}\right]=0 \\
{\left[q_{i}^{-}, q_{j}^{+}\right]=\delta_{i, j}\left(\mathbb{I}-2 K_{i}\right), \quad\left[K_{i}, q_{j}^{+}\right]=\delta_{i, j} q_{i}^{+}, \quad\left[K_{i}, q_{j}^{-}\right]=-\delta_{i, j} q_{i}^{-}}
\end{gathered}
$$

By introducing

$$
q^{-}=\sum_{i=1}^{N} q_{i}^{-}, \quad q^{+}=\sum_{i=1}^{N} q_{i}^{+}, \quad K=\sum_{i=1}^{N} K_{i}
$$

we easily obtain the commutation relations

$$
\left[q^{-}, q^{+}\right]=N \mathbb{I}-2 K, \quad\left[K, q^{+}\right]=q^{+}, \quad\left[K, q^{-}\right]=-q^{-}
$$

which generalize Equation (1). By putting

$$
a^{ \pm}=\frac{1}{\sqrt{N}} q^{ \pm}
$$

then Equation (1) can be rewritten as

$$
\left[a^{-}, a^{+}\right]=\mathbb{I}+2 \kappa K, \quad\left[K, a^{+}\right]=a^{+}, \quad\left[K, a^{-}\right]=-a^{-}
$$

where

$$
\kappa=-\frac{1}{N}
$$

so that the set $\left\{a^{-}, a^{+}, K, \mathbb{I}\right\}$ generates the generalized Weyl-Heisenberg algebra $\mathcal{A}_{\mathcal{K}}$ defined in $[17,18]$.

As a conclusion, the algebra $\mathcal{A}_{\kappa}$ can be described by a set of $N$ qubits. Here, $\kappa<0$. Therefore, according to the representation theory of $\mathcal{A}_{\kappa}$, the algebra $\mathcal{A}_{\mathcal{K}}$ admits finite-dimensional representations (for more details, see Ref. $[17,18]$ and references therein). The Dicke states (to be defined below) can serve to construct a representation of dimension $d=N+1$ of $\mathcal{A}_{\kappa}$.

\subsection{Dicke States}

The Hilbert space $\mathcal{H}_{2^{\mathrm{N}}}$ can be partitioned as

$$
\mathcal{H}_{2^{N}}=\bigoplus_{k=0}^{N} \mathcal{F}_{N, k}
$$

where the subspace $\mathcal{F}_{N, k}$, of dimension $C_{N}^{k}$, is spanned by the orthonormal set

$$
S_{N, k}=\left\{\left|\eta_{1} \eta_{2} \cdots \eta_{N}\right\rangle: \eta_{1}+\eta_{2}+\cdots+\eta_{N}=k\right\}
$$

where in each vector

$$
\left|\eta_{1} \eta_{2} \cdots \eta_{N}\right\rangle=\left|\eta_{1}\right\rangle \otimes\left|\eta_{2}\right\rangle \otimes \cdots \otimes\left|\eta_{N}\right\rangle, \quad \eta_{i}=0,1, \quad i=1,2, \cdots, N
$$

of $S_{N, k}$ there are $N-k$ qubits $|0\rangle$ and $k$ qubits $|1\rangle$ (i.e., $N-k+k=N$ qubits in all).

The Dicke state $|N ; k\rangle$ is the normalized symmetrical superposition [16,19-21]

$$
|N ; k\rangle=\left(C_{N}^{k}\right)^{-\frac{1}{2}} \sum_{|x\rangle \in S_{N, k}}|x\rangle
$$


where the summation over $|x\rangle$ is to extended over all the states of $S_{N, k}$. Note that

$$
\langle N ; k \mid N ; \ell\rangle=\delta_{k, \ell}, \quad k, \ell=0,1, \cdots, N
$$

so that the set

$$
\{|N ; k\rangle: k=0,1, \cdots, N\}
$$

of the $N+1$ symmetrical vectors $|N ; k\rangle$ constitutes an orthonormal basis for a subspace of $\mathcal{H}_{2^{N}}$; let us denote as $\mathcal{G}_{d}$ this subspace of dimension $d=N+1$.

Any normalized vector

$$
\left|\psi_{d}\right\rangle=c_{0}|N ; 0\rangle+c_{1}|N ; 1\rangle+\cdots+c_{N}|N ; N\rangle, \quad N=d-1, \quad \sum_{k=0}^{N}\left|c_{k}\right|^{2}=1, \quad c_{k} \in \mathbb{C}
$$

of $\mathcal{G}_{d}$ is a qudit expressed in terms of $N$ qubits. Each state $\left|\psi_{d}\right\rangle$ is a basis vector for the symmetric irreducible representation $[N]$ of the permutation group $S_{N}$. When $N \rightarrow \infty$, a convenient limiting procedure shows that the space $\mathcal{G}_{\infty}$ corresponds to the space of the states of the harmonic oscillator (for $N$ going to infinity, then the generalized Weyl-Heisenberg algebra $\mathcal{A}_{-\frac{1}{N}}$ goes to the harmonic oscillator algebra).

As a non-trivial example, it can be verified that the 6 vectors

$$
\begin{aligned}
|5 ; 0\rangle & =|00000\rangle \\
|5 ; 1\rangle & =\frac{1}{\sqrt{5}}(|00001\rangle+|00010\rangle+|00100\rangle+|01000\rangle+|10000\rangle) \\
|5 ; 2\rangle & =\frac{1}{\sqrt{10}}(|00011\rangle+|00101\rangle+|00110\rangle+|01001\rangle+|01010\rangle \\
& +|01100\rangle+|10001\rangle+|10010\rangle+|10100\rangle+|11000\rangle) \\
|5 ; 3\rangle & =\frac{1}{\sqrt{10}}(|00111\rangle+|01011\rangle+|01101\rangle+|01110\rangle+|10011\rangle \\
& +|10101\rangle+|10110\rangle+|11001\rangle+|11010\rangle+|11100\rangle) \\
|5 ; 4\rangle & =\frac{1}{\sqrt{5}}(|01111\rangle+|10111\rangle+|11011\rangle+|11101\rangle+|11110\rangle) \\
|5 ; 5\rangle & =|11111\rangle
\end{aligned}
$$

form an orthonormal basis for the space $\mathcal{G}_{6}$.

\subsection{Majorana States}

By using the spin $\frac{1}{2}$-spin coherent states (the so-called $\mathrm{SU}(2)$ coherent states)

$$
\left|z_{i}\right\rangle=N_{i}\left(|0\rangle+z_{i}|1\rangle\right), \quad N_{i}=\frac{1}{\sqrt{1+\bar{z}_{i} z_{i}}}, \quad i=1,2, \cdots, N
$$

the state $\left|\psi_{d}\right\rangle$ in (2) can be rewritten in the Majorana representation [22]. Note that, when necessary, the state $\left|z_{i}\right\rangle$ shall be denoted as $\left|z_{i}\right\rangle_{M}$ in order to avoid a confusion with the qubit $|1\rangle$; the state $\left|z_{i}=0\right\rangle_{M}$ is identical to the qubit $|0\rangle$ but the limit state $\left|z_{i}\right\rangle_{M}$ with $\left|z_{i}\right|=\infty$ corresponds to the qubit $|1\rangle$ while the state $\left|z_{i}=1\right\rangle_{M}$ is nothing but the qubit $\frac{1}{\sqrt{2}}(|0\rangle+|1\rangle)$. In the Majorana representation and in view of the invariance of $\left|\psi_{d}\right\rangle$ over $S_{N}$, the state $\left|\psi_{d}\right\rangle$ takes the form

$$
\left|\psi_{d}\right\rangle=\mathcal{N}_{d} \sum_{\sigma \in S_{N}} \sigma\left(\left|z_{1}\right\rangle \otimes\left|z_{2}\right\rangle \otimes \cdots \otimes\left|z_{N}\right\rangle\right)
$$


where the summation over $\sigma$ runs on all the permutations of $S_{N}$ and $\mathcal{N}_{d}$ is a normalization factor given by

$$
\left|\mathcal{N}_{d}\right|^{-2}=N ! \sum_{\sigma \in S_{N}} \prod_{i=1}^{N}\left\langle z_{i} \mid z_{\sigma(i)}\right\rangle=N ! \frac{1}{\prod_{j=1}^{N}\left(1+\bar{z}_{j} z_{j}\right)} \sum_{\sigma \in S_{N}} \prod_{i=1}^{N}\left(1+\bar{z}_{i} z_{\sigma(i)}\right) .
$$

The complex numbers $z_{i}$ for $i=1,2, \cdots, N$ are the roots (called Majorana roots or Majorana stars) of the $\mathrm{d}^{\prime}$ 'Alembert equation of degree $N\left(\right.$ for $c_{0} \neq 0$ )

$$
\sum_{k=0}^{N}(-1)^{k} \sqrt{\frac{N !}{k !(N-k) !}} c_{k} z^{N-k}=0
$$

in terms of the coefficients $c_{k}$ for $k=1,2, \cdots, N$. Conversely, the coefficients $c_{k}$ follow from

$$
c_{k}=N ! N_{1} N_{2} \cdots N_{N} \mathcal{N}_{d} \sqrt{\frac{k !(N-k) !}{N !}} s_{k}\left(z_{1} z_{2} \cdots z_{N}\right)
$$

where

$$
s_{0}\left(z_{1} z_{2} \cdots z_{N}\right)=1, \quad s_{k}\left(z_{1} z_{2} \cdots z_{N}\right)=\sum_{1 \leq i_{1}<i_{2}<\cdots<i_{k} \leq N} z_{i_{1}} z_{i_{2}} \cdots z_{i_{k^{\prime}}} \quad k=1,2, \cdots, N .
$$

In the case where the solutions of (4) are degenerate, i.e.,

$$
z=z_{1}=z_{2}=\cdots=z_{N}
$$

the state (3) becomes the completely separable state

$$
\left|\psi_{d}\right\rangle=|z\rangle \otimes|z\rangle \otimes \cdots \otimes|z\rangle=\frac{1}{(1+\bar{z} z)^{\frac{N}{2}}} \sum_{k=0}^{N} \sqrt{\frac{N !}{k !(N-k) !}} z^{k}|N ; k\rangle
$$

which is a Perelomov SU(2) coherent state.

\subsection{Procedures for Calculating Majorana States}

Given the state $\left|\psi_{d}\right\rangle$ in the form (2) with $c_{0} \neq 0$, the Majorana stars $z_{i}(i=1,2, \cdots, N)$ can be calculated from Equation (4). In the case where $c_{k}=0$ for $k=0,1, \cdots, k_{\max }$ (with $k_{\max } \leq N-1$ ), part of the Majorana stars $z_{i}$ can be calculated from (4) and part from the zeros of the Bargmann function associated with the state $\left|\psi_{d}\right\rangle$ (see Ref. [16]); in this case, Equations (5) and (6) can also be very useful for determining the Majorana stars $z_{i}$ (or checking the obtained values for $z_{i}$ ).

\section{The Perma-Concurrence}

\subsection{Expressions and Properties of the Perma-Concurrence}

We are now in a position to investigate the parameter $P_{d}$ associated with the qudit $\left|\psi_{d}\right\rangle$, or more precisely $P_{d}\left(\psi_{d}\right)$, introduced in Ref. [16] via

$$
P_{d}=\frac{1}{N !} \operatorname{perm}\left(A_{N}\right)
$$

where perm $\left(A_{N}\right)$ is the permanent of the matrix $A_{N}$, of dimension $N \times N$, with elements

$$
\left(A_{N}\right)_{i j}=\left\langle z_{i} \mid z_{j}\right\rangle=\frac{1+\bar{z}_{i} z_{j}}{\sqrt{\left(1+\left|z_{i}\right|^{2}\right)\left(1+\left|z_{j}\right|^{2}\right)}}
$$


for $i, j=1,2, \cdots, N$. A simple development of $\operatorname{perm}\left(A_{N}\right)$

$$
\operatorname{perm}\left(A_{N}\right)=\sum_{\sigma \in S_{N}} \prod_{i=1}^{N}\left\langle z_{i} \mid z_{\sigma(i)}\right\rangle=\frac{1}{\prod_{j=1}^{N}\left(1+\bar{z}_{j} z_{j}\right)} \sum_{\sigma \in S_{N}} \prod_{i=1}^{N}\left(1+\bar{z}_{i} z_{\sigma(i)}\right)
$$

that gives

$$
\operatorname{perm}\left(A_{N}\right)=\frac{1}{N !}\left|\mathcal{N}_{d}\right|^{-2}
$$

and finally we have

$$
P_{d}=\frac{1}{\left(N !\left|\mathcal{N}_{d}\right|\right)^{2}}
$$

a very useful relation for calculating $P_{d}$.

In the case where $d=3$, the parameter $P_{d}$ is an alternative to the parameter $C$, called concurrence, for symmetric two-qubit states (see Section 3.3 too). For $d$ arbitrary, the parameter $P_{d}\left(\psi_{d}\right)$, called perma-concurrence, can serve as a measure of the entanglement of the totally symmetric $N$-qubit state $\left|\psi_{d}\right\rangle$. The range of variations of $P_{d}$ is

$$
\frac{1}{2^{N-1}} \leq P_{d} \leq 1, \quad N=d-1
$$

(the range of variations $\frac{1}{N} \leq P_{d} \leq 1$ mentioned in [16] does not include maximally entangled states, except for $N=2$ ). The upper limit $P_{d}=1$ corresponds to separable states for which all the Majorana roots (7) are identical while the lower limit $P_{d}=\frac{1}{2^{N-1}}$ corresponds to maximally entangled states.

As an important property, it can be proved that

$$
P_{d}\left(\left|\psi_{d}\right\rangle\right)=P_{d}\left(\left|\varphi_{d}\right\rangle\right)
$$

where the qudit $\left|\varphi_{d}\right\rangle$ is deduced from the qudit $\left|\psi_{d}\right\rangle$ by interchanging the qubits $|0\rangle$ and $|1\rangle$ in $\left|\psi_{d}\right\rangle$.

Another useful expresion of $P_{d}$ can be obtained by introducing the inner products $n_{i} \cdot n_{j}(i, j=$ $1,2, \cdots, N)$, where the unit vectors $n_{i}$ and $n_{j}$ of $\mathbb{R}^{3}$ are defined through

$$
n_{k}=\left(\frac{z_{k}+\bar{z}_{k}}{1+z_{k} \bar{z}_{k}},-\mathrm{i} \frac{z_{k}-\bar{z}_{k}}{1+z_{k} \bar{z}_{k}}, \frac{1-z_{k} \bar{z}_{k}}{1+z_{k} \bar{z}_{k}}\right)
$$

with $\mathrm{i}=\sqrt{-1}$. Indeed, we have

$$
n_{i} \cdot n_{j}=n_{j} \cdot n_{i}=2\left|\left\langle z_{i} \mid z_{j}\right\rangle\right|^{2}-1 \Leftrightarrow\left|\left\langle z_{i} \mid z_{j}\right\rangle\right|^{2}=\frac{1}{2}\left(1+n_{i} \cdot n_{j}\right)
$$

that makes it possible to rewrite (10) in terms of $n_{i} \cdot n_{j}$ (note that $-1 \leq n_{i} \cdot n_{j} \leq 1 \Leftrightarrow 0 \leq\left|\left\langle z_{i} \mid z_{j}\right\rangle\right|^{2} \leq 1$ ).

\subsection{Procedures for Calculating the Perma-Concurrence}

For a given expression of $\left|\psi_{d}\right\rangle$ in terms of Dicke states, once all the necessary Majorana stars are calculated, the corresponding value of $P_{d}$ can be obtained from Equations (8)-(10). Another way to obtain the value of $P_{d}$ is to use (11) where $\mathcal{N}_{d}$ follows with the help of (5) and (6).

We continue with two illustrative calculations of $P_{d}$ : one for which Equation (4) is sufficient for calculating all the necessary Majorana stars and another one for which (4) allows obtaining only a part of the necessary Majorana stars. 


\subsubsection{Example 1}

The Majorana stars for the state

$$
\left|\psi_{5}\right\rangle=\sqrt{\frac{6}{3931}}\left(|4 ; 0\rangle-|4 ; 1\rangle-\frac{13}{\sqrt{6}}|4 ; 2\rangle+7|4 ; 3\rangle+24|4 ; 4\rangle\right)
$$

or

$$
\begin{aligned}
\left|\psi_{5}\right\rangle & =\sqrt{\frac{6}{3931}}\left[|0000\rangle-\frac{1}{2}(|0001\rangle+|0010\rangle+|0100\rangle+|1000\rangle)\right. \\
& -\frac{13}{6}(|0011\rangle+|0101\rangle+|0110\rangle+|1001\rangle+|1010\rangle+|1100\rangle) \\
& \left.+\frac{7}{2}(|0111\rangle+|1011\rangle+|1101\rangle+|1110\rangle)+24|1111\rangle\right]
\end{aligned}
$$

can be derived by introducing the coefficients

$$
c_{0}=\sqrt{\frac{6}{3931}}, \quad c_{1}=-c_{0}, \quad c_{2}=-\frac{13}{\sqrt{6}} c_{0}, \quad c_{3}=7 c_{0}, \quad c_{4}=24 c_{0}
$$

into Equation (4) for $N=4$. This leads to

$$
z^{4}+2 z^{3}-13 z^{2}-14 z+24=0
$$

that admits the solutions

$$
z_{1}=-4, \quad z_{2}=-2, \quad z_{3}=1, \quad z_{4}=3
$$

from which the inner products $\left\langle z_{i} \mid z_{j}\right\rangle$ with $i, j=1,2,3,4$ can be straightforwardly calculated. The value of $P_{5}$ follows from the application of (8)-(10). This leads to

$$
P_{5}=\frac{3931}{2^{3} \times 3 \times 5^{2} \times 17}
$$

in accordance with $\frac{1}{8} \leq P_{5} \leq 1$. Remark that $P_{5}$ also follows from (11) where $\mathcal{N}_{d}$ can be deduced from the coefficient $c_{0}$ given by (5). The result for $P_{5}$ shows that $\left|\psi_{5}\right\rangle$ is an entangled state but not a maximally entangled state.

\subsubsection{Example 2}

For the state

$$
\left|\psi_{4}\right\rangle=\frac{1}{2}(\sqrt{3}|3 ; 1\rangle+|3 ; 3\rangle)=\frac{1}{2}(|001\rangle+|010\rangle+|100\rangle+|111\rangle)
$$

the a priori fourth-degree d'Alembert equation, see (4), is reduced to the equation of degree 2

$$
3 z^{2}+1=0
$$

that yields the two Majorana stars

$$
z_{1}=\frac{\mathrm{i}}{\sqrt{3}}, \quad z_{2}=-\frac{\mathrm{i}}{\sqrt{3}}
$$

while the third Majorana star $z_{3}$ is such that

$$
\left|z_{3}\right|=\infty
$$


corresponding to the zero of the Bargmann function related to the state $\left|\psi_{4}\right\rangle$ (see Ref. [16]). From Equation (3), it follows that $\left|\psi_{4}\right\rangle$ can be rewritten as

$$
\left|\psi_{4}\right\rangle=\mathcal{N}_{4} \sum_{\sigma \in S_{3}} \sigma\left(\left|z_{1}\right\rangle_{M} \otimes\left|z_{2}\right\rangle_{M} \otimes\left|z_{3}\right\rangle_{M}\right)
$$

where

$$
\left|z_{1}\right\rangle_{M}=\frac{1}{2}(\sqrt{3}|0\rangle+\mathrm{i}|1\rangle), \quad\left|z_{2}\right\rangle_{M}=\frac{1}{2}(\sqrt{3}|0\rangle-\mathrm{i}|1\rangle), \quad\left|z_{3}\right\rangle_{M}=|1\rangle .
$$

The overlaps between the three states $\left|z_{i}\right\rangle_{M}$ are given by

$$
{ }_{M}\left\langle z_{1} \mid z_{2}\right\rangle_{M}=\frac{1}{2}, \quad{ }_{M}\left\langle z_{1} \mid z_{3}\right\rangle_{M}=-\frac{\mathrm{i}}{2}, \quad{ }_{M}\left\langle z_{2} \mid z_{3}\right\rangle_{M}=\frac{\mathrm{i}}{2}
$$

so that the $3 \times 3$ matrix $A_{3}$ is

$$
A_{3}=\left(\begin{array}{ccc}
1 & \frac{1}{2} & -\frac{\mathrm{i}}{2} \\
\frac{1}{2} & 1 & \frac{\mathrm{i}}{2} \\
\frac{\mathrm{i}}{2} & -\frac{\mathrm{i}}{2} & 1
\end{array}\right)
$$

cf. Equation (9). The calculation of the permanent of $A_{3}$ yields perm $\left(A_{3}\right)=\frac{3}{2}$. Finally, Equation (8) leads to $P_{4}=\frac{1}{4}$, a result showing that the 3 -qubit state $\left|\psi_{4}\right\rangle$ is maximally entangled.

3.3. Perma-Cncurrence for $N=2(d=3)$

Given the symmetric two-qubit state

$$
\left|\psi_{3}\right\rangle=c_{0}|2 ; 0\rangle+c_{1}|2 ; 1\rangle+c_{2}|2 ; 2\rangle, \quad\left|c_{0}\right|^{2}+\left|c_{1}\right|^{2}+\left|c_{2}\right|^{2}=1
$$

it is easy to show that $\left|\psi_{3}\right\rangle$ can be rewritten as

$$
\left|\psi_{3}\right\rangle=\frac{1}{2 \sqrt{P_{3}}}\left(\left|z_{1}\right\rangle \otimes\left|z_{2}\right\rangle+\left|z_{2}\right\rangle \otimes\left|z_{1}\right\rangle\right)
$$

with

$$
P_{3}=\frac{1}{2} \operatorname{perm}\left(A_{2}\right)=\frac{1}{2}\left(1+\left|\left\langle z_{1} \mid z_{2}\right\rangle\right|^{2}\right)
$$

where the Majorana stars $z_{1}$ and $z_{2}$ can be determined from the procedures in Section 2.4 with

$$
c_{0} z^{2}-\sqrt{2} c_{1} z+c_{2}=0
$$

cf. Equation (4) for $N=2$. The perma-concurrence $P_{3}$ reads

$$
P_{3}=\frac{1}{4}\left(3+n_{1} \cdot n_{2}\right)
$$

in terms of the units vectors $n_{1}$ and $n_{2}$.

It can be shown that

$$
\left|\left\langle z_{1} \mid z_{2}\right\rangle\right|^{2}=\frac{1-C}{1+C}
$$

where $C$ is the concurrence

$$
C=\left|c_{1}^{2}-2 c_{0} c_{2}\right|
$$


so that

$$
P_{3}=\frac{1}{1+C} \Leftrightarrow C=\frac{1}{P_{3}}-1
$$

and the range of variations of $P_{3}$ and $C$ are clearly

$$
\frac{1}{2} \leq P_{3} \leq 1 \Leftrightarrow 1 \geq C \geq 0 .
$$

Equation (14) shows that the situation where $C=0 \Leftrightarrow P_{3}=1$ characterizes a non entangled or separable state $\left|\psi_{3}\right\rangle$; in this case $\left\langle z_{1} \mid z_{2}\right\rangle=1 \Leftrightarrow z_{1}=z_{2} \equiv z$ and $\left|\psi_{3}\right\rangle=|z\rangle \otimes|z\rangle$. Furthermore, the situation $C=1 \Leftrightarrow P_{3}=\frac{1}{2}$ describes a maximally entangled state $\left|\psi_{3}\right\rangle$; in this case $\left\langle z_{1} \mid z_{2}\right\rangle=0 \Leftrightarrow$ $z_{1}=-\frac{1}{z_{2}} \equiv z$ and $\left|\psi_{3}\right\rangle=|z\rangle \otimes\left|-\frac{1}{\bar{z}}\right\rangle$.

As an illustration, Table 1 lists the values of the perma-concurrence $P_{3}$ and the concurrence $C$ for some states $\left|\psi_{3}\right\rangle$.

Table 1. The perma-concurrence $P_{3}$ and the concurrence $C$ for some states $c_{0}|00\rangle+c_{1} \frac{|01\rangle+|10\rangle}{\sqrt{2}}+c_{2}|11\rangle$. The Majorana state vectors $\left|z_{1}\right\rangle_{M}$ and $\left|z_{2}\right\rangle_{M}$ are expressed in terms of the qubits $|0\rangle$ and $|1\rangle$. The first three states are the maximally entangled symmetric Bell states (with $P_{3}=\frac{1}{2}$ ) and the last two states are separable symmetric states (with $P_{3}=1$ ); the value of $P_{3}$ for the other states interpolates between $\frac{1}{2}$ and 1.

\begin{tabular}{ccccccccc}
\hline$c_{0}$ & $c_{1}$ & $c_{2}$ & $z_{1}$ & $\left|z_{1}\right\rangle_{M}$ & $z_{2}$ & $\left|z_{2}\right\rangle_{M}$ & $\boldsymbol{P}_{3}$ & $C$ \\
\hline$\frac{1}{\sqrt{2}}$ & 0 & $\frac{1}{\sqrt{2}}$ & $\mathrm{i}$ & $\frac{|0\rangle+\mathrm{i}|1\rangle}{\sqrt{2}}$ & $-\mathrm{i}$ & $\frac{|0\rangle-\mathrm{i}|1\rangle}{\sqrt{2}}$ & $\frac{1}{2}$ & 1 \\
\hline$\frac{1}{\sqrt{2}}$ & 0 & $-\frac{1}{\sqrt{2}}$ & 1 & $\frac{|0\rangle+|1\rangle}{\sqrt{2}}$ & -1 & $\frac{|0\rangle-|1\rangle}{\sqrt{2}}$ & $\frac{1}{2}$ & 1 \\
\hline 0 & 1 & 0 & 0 & $|0\rangle$ & $\left|z_{2}\right|=\infty$ & $|1\rangle$ & $\frac{1}{2}$ & 1 \\
\hline 0 & $c_{1} \neq 0$ & $c_{2}$ & $\frac{c_{2}}{\sqrt{2} c_{1}}$ & $\frac{|0\rangle+z_{1}|1\rangle}{\sqrt{1+\left|z_{1}\right|^{2}}}$ & $\left|z_{2}\right|=\infty$ & $|1\rangle$ & $\frac{1}{1+\left|c_{1}\right|^{2}}$ & $\left|c_{1}\right|^{2}$ \\
\hline$\frac{1}{2 \sqrt{2}}$ & $\frac{\sqrt{2}}{4}$ & $-\frac{\sqrt{3}}{2}$ & $\frac{1+\sqrt{1+2 \sqrt{6}}}{\sqrt{2}}$ & $\frac{|0\rangle+z_{1}|1\rangle}{\sqrt{1+\left|z_{1}\right|^{2}}}$ & $\frac{1-\sqrt{1+2 \sqrt{6}}}{\sqrt{2}}$ & $\frac{|0\rangle+z_{2}|1\rangle}{\sqrt{1+\left|z_{2}\right|^{2}}}$ & $\frac{8}{57}(9-2 \sqrt{6})$ & $\frac{1+2 \sqrt{6}}{8}$ \\
\hline 0 & $\frac{1}{\sqrt{2}}$ & $\frac{1}{\sqrt{2}}$ & $\frac{1}{\sqrt{2}}$ & $\frac{\sqrt{2}|0\rangle+|1\rangle}{\sqrt{3}}$ & $\left|z_{2}\right|=\infty$ & $|1\rangle$ & $\frac{2}{3}$ & $\frac{1}{2}$ \\
\hline 0 & $\frac{1}{\sqrt{2}}$ & $-\frac{1}{\sqrt{2}}$ & $-\frac{1}{\sqrt{2}}$ & $\frac{\sqrt{2}|0\rangle-|1\rangle}{\sqrt{3}}$ & $\left|z_{2}\right|=\infty$ & $|1\rangle$ & $\frac{2}{3}$ & $\frac{1}{2}$ \\
\hline 1 & 0 & 0 & 0 & $|0\rangle$ & 0 & $|0\rangle$ & 1 & 0 \\
\hline 0 & 0 & 1 & $\left|z_{1}\right|=\infty$ & $|1\rangle$ & $\left|z_{2}\right|=\infty$ & $|1\rangle$ & 1 & 0 \\
\hline
\end{tabular}

\subsection{Perma-Concurrence for $N=3(d=4)$}

For $N=3$, the Majorana stars $z_{i}(i=1,2,3)$ follow from the procedures in Section 2.4 used in conjonction with the cubic equation

$$
c_{0} z^{3}-\sqrt{3} c_{1} z^{2}+\sqrt{3} c_{2} z-c_{3}=0
$$

cf. (4). Then, Equations (8)-(10) lead to

$$
P_{4}=\frac{1}{6}\left(1+\left|\left\langle z_{1} \mid z_{2}\right\rangle\right|^{2}+\left|\left\langle z_{2} \mid z_{3}\right\rangle\right|^{2}+\left|\left\langle z_{3} \mid z_{1}\right\rangle\right|^{2}+\left\langle z_{1} \mid z_{2}\right\rangle\left\langle z_{2} \mid z_{3}\right\rangle\left\langle z_{3} \mid z_{1}\right\rangle+\left\langle z_{1} \mid z_{3}\right\rangle\left\langle z_{3} \mid z_{2}\right\rangle\left\langle z_{2} \mid z_{1}\right\rangle\right)
$$

which can be simplified as

$$
P_{4}=\frac{1}{3}\left(\left|\left\langle z_{1} \mid z_{2}\right\rangle\right|^{2}+\left|\left\langle z_{2} \mid z_{3}\right\rangle\right|^{2}+\left|\left\langle z_{3} \mid z_{1}\right\rangle\right|^{2}\right)
$$


which in turn yields

$$
P_{4}=\frac{1}{6}\left(3+n_{1} \cdot n_{2}+n_{2} \cdot n_{3}+n_{3} \cdot n_{1}\right)
$$

owing to Equation (12).

We give in Table 2 some examples of states $\left|\psi_{4}\right\rangle$ for which $P_{4}$ goes from $\frac{1}{4}$ (for maximally entangled states) to 1 (for separable states).

Table 2. The perma-concurrence $P_{4}$ for some states $c_{0}|000\rangle+c_{1} \frac{|001\rangle+|010\rangle+|100\rangle}{\sqrt{3}}+c_{2} \frac{|011\rangle+|101\rangle+|110\rangle}{\sqrt{3}}+$ $c_{3}|111\rangle$. The parameters $\omega, a$ and $b$ are $\omega=\exp \left(\mathrm{i} \frac{2 \pi}{3}\right), a=\sqrt[3]{4}$ and $b=\sqrt[3]{2}$. The first three states are maximally entangled symmetric states of GHZ type (with $P_{4}=\frac{1}{4}$ ) and the last two states are separable symmetric states (with $P_{4}=1$ ); the value of $P_{4}$ for the other states interpolates between $\frac{1}{4}$ and 1 .

\begin{tabular}{cccccccc}
\hline$c_{0}$ & $c_{1}$ & $c_{2}$ & $c_{3}$ & $z_{1}$ & $z_{2}$ & $z_{3}$ & $\boldsymbol{P}_{4}$ \\
\hline$\frac{1}{\sqrt{2}}$ & 0 & 0 & $\frac{1}{\sqrt{2}}$ & 1 & $\omega$ & $\omega^{2}$ & $\frac{1}{4}$ \\
\hline$\frac{1}{2}$ & 0 & $\frac{\sqrt{3}}{2}$ & 0 & 0 & $\mathrm{i} \sqrt{3}$ & $-\mathrm{i} \sqrt{3}$ & $\frac{1}{4}$ \\
\hline 0 & $\frac{\sqrt{3}}{2}$ & 0 & $\frac{1}{2}$ & $\frac{\mathrm{i}}{\sqrt{3}}$ & $-\frac{\mathrm{i}}{\sqrt{3}}$ & $\left|z_{3}\right|=\infty$ & $\frac{1}{4}$ \\
\hline$\frac{1}{\sqrt{8}}$ & $\frac{\sqrt{3}}{\sqrt{8}}$ & $-\frac{\sqrt{3}}{\sqrt{8}}$ & $\frac{1}{\sqrt{8}}$ & $a+b+1$ & $\frac{2-a-b}{2}+\mathrm{i} \sqrt{3} \frac{a-b}{2}$ & $\frac{2-a-b}{2}-\mathrm{i} \sqrt{3} \frac{a-b}{2}$ & $\frac{4 b}{6+3 a+4 b}$ \\
\hline 0 & 1 & 0 & 0 & 0 & 0 & $\left|z_{3}\right|=\infty$ & $\frac{1}{3}$ \\
\hline$\frac{1}{2}$ & $\frac{\sqrt{3}}{2}$ & 0 & 0 & 0 & 0 & 3 & $\frac{2}{5}$ \\
\hline 0 & 0 & $\frac{\sqrt{3}}{2}$ & $\frac{1}{2}$ & $\frac{1}{3}$ & $\left|z_{2}\right|=\infty$ & $\left|z_{3}\right|=\infty$ & $\frac{2}{5}$ \\
\hline 1 & 0 & 0 & 0 & 0 & 0 & 0 & 1 \\
\hline 0 & 0 & 0 & 1 & $\left|z_{1}\right|=\infty$ & $\left|z_{2}\right|=\infty$ & $\left|z_{3}\right|=\infty$ & 1 \\
\hline
\end{tabular}

\subsection{Perma-Concurrence for $N=4(d=5)$}

From Equations (4), (8)-(10) and (12), the basic ingredients for dealing with the case $N=4$ are the following:

$$
c_{0} z^{4}-2 c_{1} z^{3}+\sqrt{6} c_{2} z^{2}-2 c_{3} z+c_{4}=0
$$

and

$$
\begin{aligned}
P_{5} & =\frac{1}{12}\left[\left|\left\langle z_{1} \mid z_{2}\right\rangle\right|^{2}\left|\left\langle z_{3} \mid z_{4}\right\rangle\right|^{2}+\left|\left\langle z_{1} \mid z_{3}\right\rangle\right|^{2}\left|\left\langle z_{2} \mid z_{4}\right\rangle\right|^{2}+\left|\left\langle z_{1} \mid z_{4}\right\rangle\right|^{2}\left|\left\langle z_{2} \mid z_{3}\right\rangle\right|^{2}\right. \\
& \left.+2\left(\left|\left\langle z_{1} \mid z_{2}\right\rangle\right|^{2}+\left|\left\langle z_{1} \mid z_{3}\right\rangle\right|^{2}+\left|\left\langle z_{1} \mid z_{4}\right\rangle\right|^{2}+\left|\left\langle z_{2} \mid z_{3}\right\rangle\right|^{2}+\left|\left\langle z_{2} \mid z_{4}\right\rangle\right|^{2}+\left|\left\langle z_{3} \mid z_{4}\right\rangle\right|^{2}\right)-3\right]
\end{aligned}
$$

which can be equally written as

$$
\begin{aligned}
P_{5} & =\frac{1}{48}\left[\left(n_{1} \cdot n_{2}\right)\left(n_{3} \cdot n_{4}\right)+\left(n_{1} \cdot n_{3}\right)\left(n_{2} \cdot n_{4}\right)+\left(n_{1} \cdot n_{4}\right)\left(n_{2} \cdot n_{3}\right)\right. \\
& \left.+5\left(n_{1} \cdot n_{2}+n_{1} \cdot n_{3}+n_{1} \cdot n_{4}+n_{2} \cdot n_{3}+n_{2} \cdot n_{4}+n_{3} \cdot n_{4}\right)+15\right]
\end{aligned}
$$

in terms of the unit vectors $n_{i}$ with $i=1,2,3,4$.

3.6. Perma-Concurrence for $N=5(d=6)$

The calculation of $P_{6}$ gives

$$
P_{6}=\frac{1}{80}\left(15+5 P_{6,1}+P_{6,2}\right)
$$


where

$$
P_{6,1}=n_{1} \cdot n_{2}+n_{1} \cdot n_{3}+n_{1} \cdot n_{4}+n_{1} \cdot n_{5}+n_{2} \cdot n_{3}+n_{2} \cdot n_{4}+n_{2} \cdot n_{5}+n_{3} \cdot n_{4}+n_{3} \cdot n_{5}+n_{4} \cdot n_{5}
$$

and

$$
\begin{aligned}
P_{6,2} & =\left(n_{1} \cdot n_{2}\right)\left(n_{3} \cdot n_{4}\right)+\left(n_{1} \cdot n_{2}\right)\left(n_{3} \cdot n_{5}\right)+\left(n_{1} \cdot n_{2}\right)\left(n_{4} \cdot n_{5}\right) \\
& +\left(n_{1} \cdot n_{3}\right)\left(n_{2} \cdot n_{4}\right)+\left(n_{1} \cdot n_{3}\right)\left(n_{2} \cdot n_{5}\right)+\left(n_{1} \cdot n_{3}\right)\left(n_{4} \cdot n_{5}\right) \\
& +\left(n_{1} \cdot n_{4}\right)\left(n_{2} \cdot n_{3}\right)+\left(n_{1} \cdot n_{4}\right)\left(n_{2} \cdot n_{5}\right)+\left(n_{1} \cdot n_{4}\right)\left(n_{3} \cdot n_{5}\right) \\
& +\left(n_{1} \cdot n_{5}\right)\left(n_{2} \cdot n_{3}\right)+\left(n_{1} \cdot n_{5}\right)\left(n_{2} \cdot n_{4}\right)+\left(n_{1} \cdot n_{5}\right)\left(n_{3} \cdot n_{4}\right) \\
& +\left(n_{2} \cdot n_{3}\right)\left(n_{4} \cdot n_{5}\right)+\left(n_{2} \cdot n_{4}\right)\left(n_{3} \cdot n_{5}\right)+\left(n_{2} \cdot n_{5}\right)\left(n_{3} \cdot n_{4}\right) .
\end{aligned}
$$

\section{Perma-Concurrence for Symmetric N-Qubit States of W Type}

In this section, we consider qudits $\left|\psi_{d}\right\rangle, d=N-1$, for which

$$
\left\langle z_{1} \mid z_{2}\right\rangle=0, \quad\left\langle z_{1} \mid z_{3}\right\rangle=0, \quad \cdots, \quad\left\langle z_{1} \mid z_{N}\right\rangle=0
$$

up to an arbitrary permutation of the indices $1,2, \cdots, N$. Then, the corresponding perma-concurrence $P_{d}$ assumes the following particular expression

$$
P_{d}=\frac{1}{N !} \sum_{\sigma \in S_{N-1}} \prod_{i=2}^{N}\left\langle z_{i} \mid z_{\sigma(i)}\right\rangle
$$

where the sum on $\sigma$ and the product on $i$ are to be performed on $S_{N-1}$ (instead of $S_{N}$ ) and from 2 to $N$ (instead of 1 to $N$ ), respectively. The conditions (17) imply that

$$
z_{2}=z_{3}=\cdots=z_{N}=-\frac{1}{\bar{z}}, \quad z=z_{1}
$$

so that $P_{d}$ takes the simple form

$$
P_{d}=\frac{1}{N}=\frac{1}{d-1}
$$

a value compatible with the domain $\frac{1}{2^{N-1}} \leq P_{d} \leq 1$

Consequently, the state $\left|\psi_{d}\right\rangle$ with $P_{d}=\frac{1}{d-1}$ reads in the Majorana representation as

$$
\left|\psi_{d}\right\rangle=\frac{1}{\sqrt{N}}\left(\left|z,-\frac{1}{\bar{z}}, \cdots,-\frac{1}{\bar{z}}\right\rangle_{M}+\left|-\frac{1}{\bar{z}}, z, \cdots,-\frac{1}{\bar{z}}\right\rangle_{M}+\cdots+\left|-\frac{1}{\bar{z}^{\prime}},-\frac{1}{\bar{z}^{\prime}}, \cdots, z\right\rangle_{M}\right)
$$

which is a state of $\mathrm{W}$ type [26]. Such a general state includes the special states

$$
|\mathrm{W}\rangle_{N}=\frac{1}{\sqrt{N}}(|0,1, \cdots, 1\rangle+|1,0, \cdots, 1\rangle+\cdots+|1, \cdots, 1,0\rangle)
$$

and

$$
|\overline{\mathrm{W}}\rangle_{N}=\frac{1}{\sqrt{N}}(|1,0, \cdots, 0\rangle+|0,1, \cdots, 0\rangle+\cdots+|0, \cdots, 0,1\rangle)
$$

corresponding to $z \longrightarrow 0$ and $|z| \longrightarrow \infty$, respectively.

The entanglement (not maximum entanglement) of the general state (18) can be described geometrically in terms of unit vectors. For instance, let us consider the cases $N=2,3$ and 4 . For $N=2$, the perma-concurrence $P_{3}$ given by (13) takes its minimal value $P_{3}=\frac{1}{N}=\frac{1}{2}$ (corresponding to maximally entangled states) for $n_{1} \cdot n_{2}=-1$ showing that the two vectors $n_{1}$ and $n_{2}$ have opposite directions. For $N=3$, Equation (15) gives $P_{4}=\frac{1}{N}=\frac{1}{3}$ for entangled 3-qubit states such that 
$n_{1} \cdot n_{2}=-1$ and $n_{1} \cdot n_{3}=-1$ which imply $n_{2} \cdot n_{3}=1$; therefore, two unit vectors coincide and point in the opposite direction of the third one. For $N=4$, the value $P_{5}=\frac{1}{N}=\frac{1}{4}$ of the perma-concurrence can be obtained from Equation (16) with

$$
n_{1} \cdot n_{2}=-1, \quad n_{1} \cdot n_{3}=-1, \quad n_{1} \cdot n_{4}=-1
$$

which imply

$$
n_{2} \cdot n_{3}=1, \quad n_{2} \cdot n_{4}=1, \quad n_{3} \cdot n_{4}=1
$$

so that for entangled states with $P_{5}=\frac{1}{4}$ the unit vectors $n_{2}, n_{3}$ and $n_{4}$ are identical with an opposite direction to $n_{4}$.

\section{Perma-Concurrence for Symmetric N-Qubit States of Bell and GHZ Type}

We now consider the $\mathrm{N}$-qubit state

$$
\left|\psi_{d}\right\rangle=\frac{1}{\sqrt{2}}(|N ; 0\rangle+|N ; N\rangle)=\frac{1}{\sqrt{2}}(|00 \cdots 0\rangle+|11 \cdots 1\rangle)
$$

which corresponds to

$$
c_{1}=c_{2}=\cdots=c_{N-1}=0, \quad c_{0}=c_{N}=\frac{1}{\sqrt{2}}
$$

in (2). Hence, $\left|\psi_{d}\right\rangle$ is a Bell state for $N=2$ and a GHZ state for $N>2$. The corresponding $N$ Majorana stars $z_{k}$ are here entirely determined by

$$
z^{N}+(-1)^{N}=0
$$

that follows from (4). Thus, the Majorana roots $z_{k}$ are

$$
z_{k}=\exp \left(\mathrm{i}(2 k+1) \pi \frac{N+1}{N}\right), \quad k=1,2, \cdots, N
$$

from which we can calculate the perma-concurrence $P_{d}$. It is sufficient to use (11). The normalisation factor $\mathcal{N}_{d}$ in (11) follows from (5) with $k=0$, viz,

$$
\frac{1}{\sqrt{2}}=N ! N_{1} N_{2} \cdots N_{N} \mathcal{N}_{d}
$$

with $N_{1}=N_{2}=\cdots=N_{N}=\frac{1}{\sqrt{2}}$. This yields

$$
P_{d}=\frac{1}{2^{N-1}}=\frac{1}{2^{d-2}}
$$

which corresponds to the minimum value of $P_{d}$.

It is easy to check that the result given by Equation (21) holds for the states

$$
\left|\psi_{d}\right\rangle=\frac{1}{\sqrt{2}}(|00 \cdots 0\rangle-|11 \cdots 1\rangle)
$$

for $N \geq 2$ and

$$
\left|\psi_{3}\right\rangle=\frac{1}{\sqrt{2}}(|01\rangle+|10\rangle)
$$

for $N=2$. We thus recover that the symmetric Bell states and the GHZ states are maximally entangled.

As an example, let us consider the state 


$$
\left|\psi_{6}\right\rangle=\frac{1}{\sqrt{2}}(|00000\rangle+|11111\rangle)
$$

corresponding to $c_{0}=c_{5}=\frac{1}{\sqrt{2}}$ and $c_{1}=c_{2}=c_{3}=c_{4}=0$. The Majorana roots $z_{k}$ are the solutions of the simple equation

$$
z^{5}-1=0
$$

that gives

$$
z_{1}=\omega, \quad z_{2}=\omega^{2}, \quad z_{3}=\omega^{3}, \quad z_{4}=\omega^{4}, \quad z_{5}=\omega^{5}=1, \quad \omega=\exp \left(\frac{\mathrm{i} 2 \pi}{5}\right)
$$

in agreement with (19). The state $\left|\psi_{6}\right\rangle$ can be written

$$
\left|\psi_{6}\right\rangle=\mathcal{N}_{6} \sum_{\sigma \in S_{5}} \sigma\left(\left|z_{1}\right\rangle_{M} \otimes\left|z_{2}\right\rangle_{M} \otimes\left|z_{3}\right\rangle_{M} \otimes\left|z_{4}\right\rangle_{M} \otimes\left|z_{5}\right\rangle_{M}\right)
$$

where

$$
\left|z_{k}\right\rangle_{M}=\frac{1}{\sqrt{2}}\left(|0\rangle+\omega^{k}|1\rangle\right)
$$

for $k=1,2,3,4,5$. From a geometrical point of view, we have

$$
\left\langle z_{i} \mid z_{j}\right\rangle=\frac{1}{2}\left(1+\omega^{j-i}\right)
$$

and

$$
n_{i} \cdot n_{j}=\cos \left[(j-i) \frac{2 \pi}{5}\right]
$$

Equation (20) can be particularized as

$$
\frac{1}{\sqrt{2}}=5 !\left(\frac{1}{\sqrt{2}}\right)^{5} \mathcal{N}_{6} \Rightarrow \mathcal{N}_{6}=\frac{1}{30}
$$

and finally (11) gives $P_{6}=\frac{1}{16}$.

\section{Concluding Remarks and Prospects}

The aim of the present paper was to investigate the properties and calculation procedures of the perma-concurrence parameter $P_{d}$ defined in Ref. [16]. The parameter $P_{d}$ makes it possible to characterize the entanglement degree of a qudit $\left|\psi_{d}\right\rangle$ (in dimension $d$ ) constructed from Dicke states made of $N$-qubits with $N=d-1$. It varies from $P_{d}=\frac{1}{2^{d-2}}$ (for maximally entangled states) to $P_{d}=1$ (for non entangled states, i.e., separable states). The perma-concurrence $P_{d}$ extends to totally symmetric multi-qubit systems the notion of concurrence for two-qubit systems. Numerous examples were given in order to complete, precise and render easier the formalism developed in [16].

Due to the invariance of the Dicke states under the symmetric group $S_{N}$, the parameter $P_{d}$ works only for totally symmetric $N$-qubit states. This emphasizes the importance of $S_{N}$ in the construction of $P_{d}$. In this respect, it would be interesting to define a parameter similar to $P_{d}$ for a $N$-qubit state transforming as an arbitrary irreducible representation of $S_{N}$.

Author Contributions: The authors equally contributed to the paper. Conceptualization: M.D. and M.R.K.

Funding: This research received no external funding.

Acknowledgments: One of the authors (MD) would like to thank the hospitality and kindness of the Groupe Théorie de l'Institut de Physique Nucléaire de Lyon [renamed as Institut de Physique des 2 Infinis (IP2I) de Lyon since July 1, 2019] where this work was done. The authors are grateful to M.S. Byrd for giving their attention to two relevant papers. 
Conflicts of Interest: The authors declare no conflict of interest.

\section{References}

1. Nielsen, M.A.; Chuang I.L. Quantum Computation and Quantum Information; Cambridge University Press: Cambridge, UK, 2000.

2. Braunstein, S.L.; Kimble, H.J. Teleportation of continuous quantum variables. Phys. Rev. Lett. 1998, 80, 869-872. [CrossRef]

3. Bouwmeester, D.; Pan, J.W.; Mattle, K.; Eibl, M.; Weinfurter, H.; Zeilinger, A. Experimental quantum teleportation. Nature 1997, 390, 575-579. [CrossRef]

4. Bennett, C.H.; Brassard, G. Quantum cryptography: Public key distribution and coin tossing. In Proceedings of the IEEE International Conference on Computers, Systems and Signal Processing, Bangalore, India, 9-12 December 1984; IEEE: New York, NY, USA, 1984; p. 175.

5. Vedral, V. The role of relative entropy in quantum information theory. Rev. Mod. Phys. 2002, 74, 197. [CrossRef]

6. Horodecki, R.; Horodecki, P.; Horodecki, M.; Horodecki, K. Quantum entanglement. Rev. Mod. Phys. 2009, 81, 865. [CrossRef]

7. Gühne O.; Tóth, G. Entanglement detection. Phys. Rep. 2009, 474, 1-75. [CrossRef]

8. Modi, K.; Brodutch, A.; Cable, H.; Paterek, T.; Vedral, V. Quantum discord and other measures of quantum correlation. Rev. Mod. Phys. 2012, 84, 1655. [CrossRef]

9. Wootters, W.K. Entanglement of formation of an arbitrary state of two qubits. Phys. Rev. Lett. 1998, 80, 2245. [CrossRef]

10. Ollivier, H.; Zurek, W.H. Quantum discord: A measure of the quantumness of correlations. Phys. Rev. Lett. 2001, 88, 017901. [CrossRef] [PubMed]

11. Henderson, L.; Vedral, V. Classical, quantum and total correlations. J. Phys. A Math. Gen. 2001, $34,6899$. [CrossRef]

12. Coffman, V.; Kundu, J.; Wootters, W.K. Distributed entanglement. Phys. Rev. A 2000, 61, 052306. [CrossRef]

13. Wong A.; Christensen, N. Potential multiparticle entanglement measure. Phys. Rev. A 2001, 63, 044301. [CrossRef]

14. Li, D. Stochastic local operations and classical communication (SLOCC) and local unitary operations (LU) classifications of $\mathrm{n}$ qubits via ranks and singular values of the spin-flipping matrices. Quantum Inf. Process 2018, 17, 132. [CrossRef]

15. López-Saldívar, J.A.; Castaños, O.; Nahmad-Achar, E.; López-Peña, R.; Man'ko, M.A.; Man'ko, V.I. Geometry and entanglement of two-qubit states in the quantum probabilistic representation. Entropy 2018, 20, 630. [CrossRef]

16. Daoud, M.; Kibler, M.R. Generalized Weyl-Heisenberg Algebra, Qudit Systems and Entanglement Measure of Symmetric States via Spin Coherent States. Entropy 2018, 20, 292. [CrossRef]

17. Daoud, M.; Kibler, M.R. Phase operators, temporally stable phase states, mutually unbiased bases and exactly solvable quantum systems. J. Phys. A Math. Theor. 2010, 43, 115303. [CrossRef]

18. Daoud, M.; Kibler, M.R. Phase operators, phase states and vector phase states for $S U_{3}$ and $S U_{2,1}$. J. Math. Phys. 2011, 52, 082101. [CrossRef]

19. Dicke, R. Coherence in spontaneous radiation processes. Phys. Rev. 1954, 93, 99. [CrossRef]

20. Tóth, G. Detection of multipartite entanglement in the vicinity of symmetric Dicke states. J. Opt. Soc. Am. B 2007, 24, 275-282. [CrossRef]

21. Bergmann, M.; Gühne, O. Entanglement criteria for Dicke states. J. Phys. A Math. Theor. 2013, 46, 385304. [CrossRef]

22. Majorana, E. Atomi orientati in campo magnetico variabile. Nuovo Cimento 1932, 9, 43-50. [CrossRef]

23. Bishop, C.A.; Byrd M.S.; Wu, L.-A. Casimir invariants for systems undergoing collective motion. Phys. Rev. A 2011, 83, 062327. [CrossRef]

24. Byrd, M.S. Implications of qudit superselection rules for the theory of decoherence-free subsystems. Phys. Rev. A 2006, 73, 032330. [CrossRef] 
25. Kolenderski, P. Geometry of pure states of N spin-J system. Open Syst. Inf. Dyn. 2010, 17, 107-119. [CrossRef] 26. Dür, W.; Vidal, G.; Cirac, J.I. Three qubits can be entangled in two inequivalent ways. Phys. Rev. A 2000, 62, 062314. [CrossRef] 